\title{
EINIGE ALTNORDARABISCHE INSCHRIFTEN AUS JORDANIEN
}

\author{
ALI AL-MANASER \\ Khalili Research Centre for the Art and Material Culture of the Middle East, University of Oxford \\ 3 St. John st., Oxford OX1 2LG, United Kingdom \\ e-mail: ali.al-manaser@orinst.ox.ac.uk
}

This paper deals with new epigraphical material which was excavated in Wādī al-Hašād in Jordan. The importance of this study lies in the publication of these yet unpublished inscriptions.

Key words: Safaitic inscriptions, Early Arabian inscriptions, Jordan, Semitic linguistics.

Dieser Artikel befasst sich mit acht neuen altnordarabischen Inschriften, die im Jahr 2004 in Jordanien gefunden wurden. Neben der Veröffentlichung dieser neuen Inschriften geht der Artikel auf die Beziehung zwischen dem Fundort und den Inhalt der Inschriften ein. Zudem werden die Reiterabbildungen näher betrachtet. Außerdem beschäftigt sich dieser Beitrag mit einem neuen Stammesnamen in den safaitischen Inschriften.

Die safaitischen Inschriften sind die größte Gruppe von nordarabischen Inschriften, die bisher gefunden wurden. Dabei handelt es sich um ca. 40.000 Inschriften. Die Inschriften dieses Artikels wurden im Jahr 2004 mit 400 anderen Inschriften in einem Ort namens Wādī al-Hašād, etwa $42 \mathrm{~km}$ nordöstlich von dem Dorf aṣ-Ṣafāwī, gefunden (sehe Landkarte). Die Ortansässigen gaben dem Wadi seinen Namen aufgrund der vielen kleinen schwarzen Steine (Basaltstein), die dort zu finden sind. Alle Inschriften sind in Basaltstein eingraviert worden. 


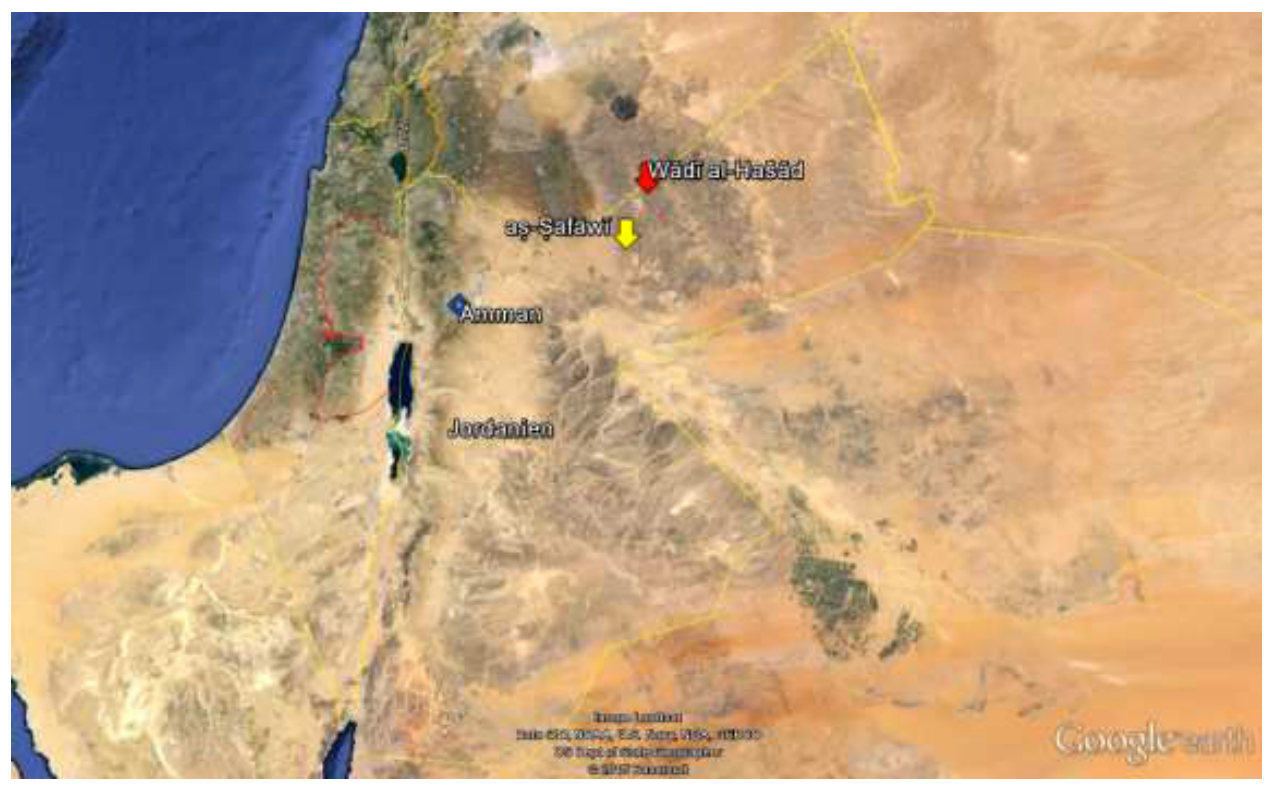

Landkarte zur geographischen Lage von Wādī al-Hašād

\section{Die Inschriften}

\section{Inschrift 1.}

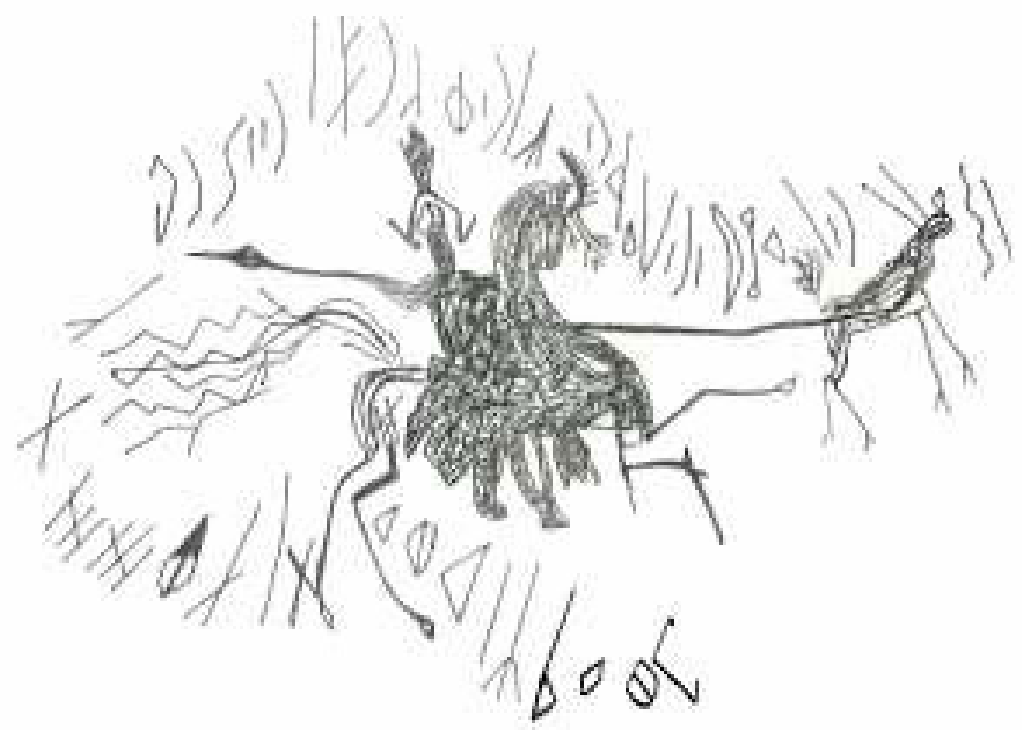



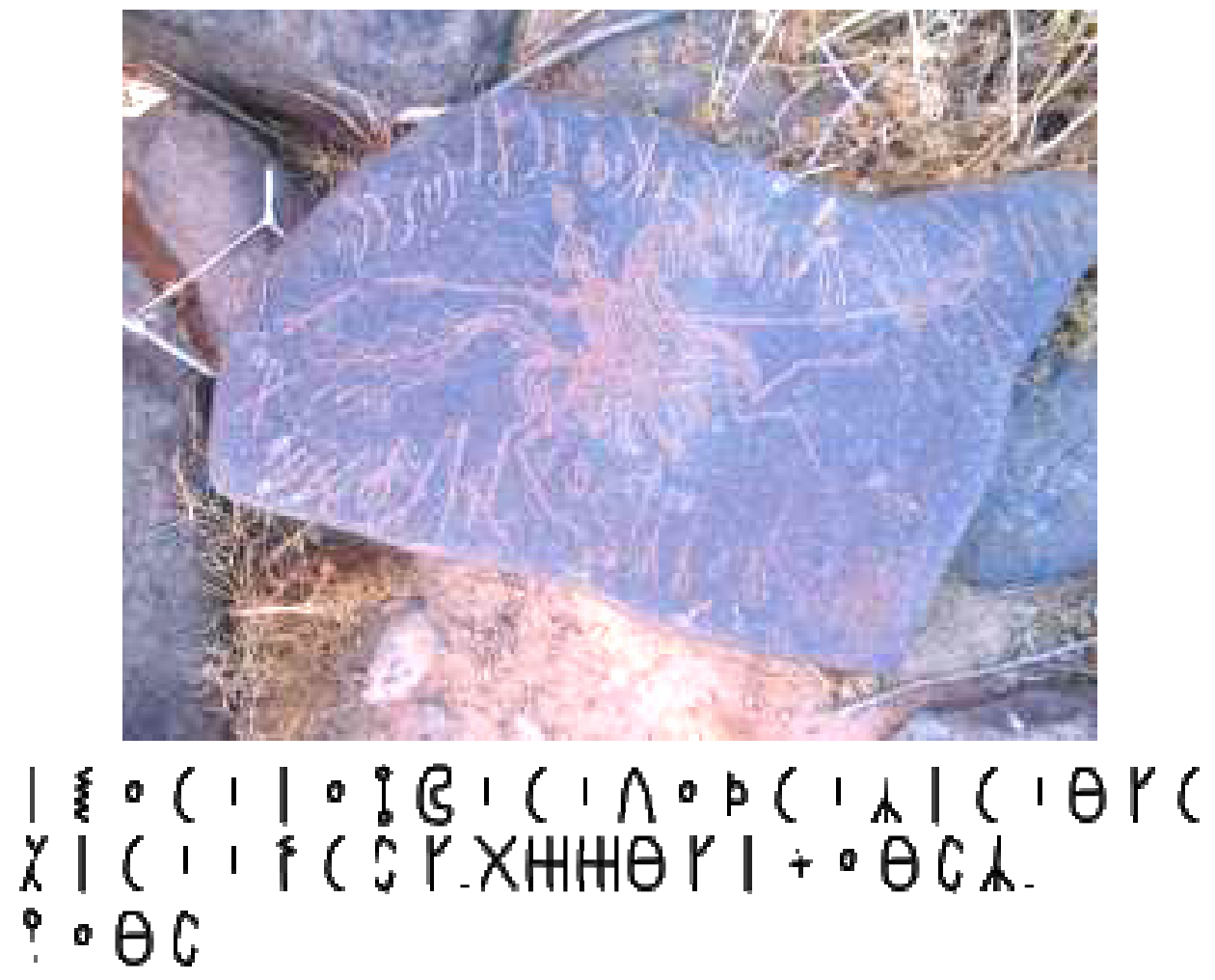

\section{Transliteration}



Dem $\mathrm{S}^{2}$ bin L'tmn bin $\mathrm{S}^{1} \mathrm{~d}$ bin $\mathrm{D} 1$ bin Whb'l bin Ng̈br (gehört) diese Zeichnug. Und o Lāt, Blindheit dem, der [diese Inschrift] auslöscht!

L: Diese Inschrift fängt wie die meisten safaitischen Inschriften mit dem Partikel $l$ an. Es wird mit „von“ oder „für“ bzw. englisch „by“ übersetzt (Al-Manaser 2008, S. 79) ${ }^{1}$.

$\mathbf{S}^{2}$ : Dieser Name wurde wahrscheinlich aus der Wurzel $\check{S} y / w^{\prime}$ „,beistehen, begleiten, sich anschließen“, šlı 'ah „Gefolgsmann, Beistand“" (Lane 1863-1893, S. 1631) abgeleitet. Er findet sich im Safaitischen (CIS 717; ISB 209; SIJ 20; HCH 22; LP 288; WH 212), $S^{2}$ '-lh im Tamūdischen (Shatnawi 2002, S. 710), $S^{2}$ und $S 2 y^{\prime}$ im Lihyanischen (Caskel 1954, S. 152), S2 'yw im Nabatäischen (Negev 1991, S. 66), Sy' und Sy't „to help“ im Palmyrenischen (Stark 1971, S. 101) und ' $l-s ̌ w$ 'im Qatabānischen (PQI 79).

${ }^{1}$ Zur weiteren Diskussion des Partikel $l$, siehe Al-Jallad (2015, S. 4-6) and Macdonald (2006, S. 294-295). 
L'țmn: Er ist vielleicht mit arabisch 'Aytūm ,stark und groß“', 'Aytūum „Elefant oder Hyäne“, 'A țamtam „Löwe“, 'Utmān „Trappenjunge“ (Lane 1863-1893, S. 1954) zu vergleichen. Der Name findet sich im Safaitischen (CIS 401; LP 322; WH 606).

Dl: Der vorliegende Name geht wahrscheinlich auf die Wurzel $D l l$, vergleiche arabisch Dill „niedrig, verächtlich sein, erniedrigen“ (Al-Manaser 2008, S. 207) zurück. Der Name $\underline{D} l$ kommt im Safaitischen (CIS 116; LP 73; SIJ 226; WH 179), im Tamūdischen (HIn 256) und im Qatabānischen (PQI 141).

$\mathbf{S}^{1}$ d: Die Deutung dieses Namen stützt sich auf die gemeinsemitische Wurzel $s$ ' $d$ „glücklich sein“, Vergleiche im Arabischen sa ida ,glücklich sein“, sa'd „Glück“, sā ‘ada „,helfen, unterstützen“ (Al-Manaser 200, S. 125). Der Name findet sich darüber hinaus bereits im Safaitischen (CIS 35; LP 82; HCH 10; ISB 113), im Tamūdischen (King 1990, S. 509), im Lihyanischen (Sima 1999, S. 76), im Minäischen (PMI 118), im Nabatäischen in den Formen $S 1$ ' $d y$, $S 1$ ' $d$ ' und $S 1$ ' $d w$ (Negev 1991, S. 65, 66), im Palmyrenischen. in den Formen $S 1^{\prime} d, S 1^{\prime} d$ ', $S 1^{\prime} d w$ und $S 1^{\prime} d y$ (Stark 1971, S. 115), im Qatabānischen (PQI 160), im Hatrenisch in der Form $S 1^{\prime} d w$ (PIH 172) und in griechisch Transkription als $\Sigma \alpha \delta \alpha, \Sigma \alpha ́ \delta \alpha$ und $\Sigma \alpha ́ \delta$ (Wuthnow 1930, S. 101, 178) nachweisen.

Whb'l: Dabei handelt es sich um einen zusammengesetzten Namen aus dem th. ' $l$ und dem Element whb, Wahb 'il „Gabe bzw. Geschenk Gottes ('il)“ (Hazim 1986, S. 136). Der Name findet sich im Safaitischen (CIS 320; LP 155; SIJ 50; HCH 110; WH 77) und im Tamūdischen (TIJ 37).

Ngbr: Bei diesem Namen handelt es sich wahrscheinlich um die Wurzel $\dot{g} b r ; \dot{g} a b a r a$ „,bleiben und gehen“, gāabir „die Vergangenheit“, gubār „Staub“ (Lane 18631893, S. 2224). Der Name findet sich darüber hinaus bereits im Safaitischen (CIS 99; LP 342; SIJ 421;WH 1770).

h: Der Artikel $h$ - im Safaitischen übernimmt manchmal jedoch die Funktion eines Demonstrativpronomens im Deutschen. Dies ist meist in Inschriften, die ein Besitzverhältnis ausdrücken, der Fall (Al-Jallad 2015, S. 16). The Definite Article h-htț: hä-hutut „,die Inschrift, die Zeichnung“ (KRS 3224).

$w a$-h-Lāt: Eine der bekanntesten safaitischen Göttinnen und die am häufigsten erwähnte Gottheit in den safaitischen Inschriften.

'wr: 'awwir „,blende“" (Voigt 1981, S. 181).

d: [li]- $\underline{d}$ Relativpronomen ,wer, den, welcher..." (Al-Jallad 2015, S. 80).

y'wr: yu 'awwiru ,zerstören, auslöschen“" (Al-Jallad 2015, S. 69).

Neben der Inschrift ist ein Reiter abgebildet, der eine Gazelle jagt. Die mit einfachen Strichen und Linien umgesetzte Zeichnung erscheint hierbei zweidimensional. Der nachoben erhobene Kopf erweckt den Anschein, als ob der Reiter in Richtung des Betrachters des Bildes blickt. Die Kopfbedeckung mit der Feder symbolisiert Macht und Reichtum und deutet wahrscheinlich darauf hin, dass es sich bei dem Reiter um eine wichtige und bedeutende Person wie etwa einen Scheich oder einen Fürst handelt. Seine linke Hand hält die Zügel seines Pferdes fest und demonstriert dadurch seine Macht über das Tier. Die rechte Hand umfasst einen Speer, der nach hinten gestreckt, das Gefühl vermittelt, die Gazelle genau in diesem Moment zu erlegen. Durch 
die Anordnung der Vorderbeine des Pferdes soll dem Betrachter das Gefühl vermittelt werden, dass sich der Reiter und das Pferd wirklich in Bewegung befinden und die Gazelle verfolgen wollen. Der Kopf des Pferdes ist mit etwas Federähnlichem geschmückt. Dies kann mit der anscheinend hohen Stellung des Reiters in Verbindung gebracht werden. Die Darstellung der Hinterbeine des Pferdes in fast physisch normaler Stellung vermittelt den Eindruck von dessen Stärke und guter Abstammung. Die Schnelligkeit des Pferdes wird durch die einfache Striche dargestellten Hufeisendes Pferdes angedeutet. Der fliegende Schweif des Pferdes soll auf seine Vortrefflichkeit hinweisen. Denn der Wert eines Pferdes ermisst sich aus der Art des Schweifes. Am Ende des Speeres ist eine Art von Kugel oder Gewicht erkennbar. Ihre Bedeutung oder Aufgabe bestand vielleicht darin, die Balance des Speeres mit der metallischen Spitze aufrechtzuerhalten. Dass dieses Gewicht mehr Kraft für den Speerbeim Stoß in die Gazelle erzeugte oder erzeugen sollte, erscheint weniger wahrscheinlich. Damals verfügte man vermutlich noch nicht über das Wissen, um solche physikalischen Zusammenhänge zu erfassen.

\section{Inschrift 2.}

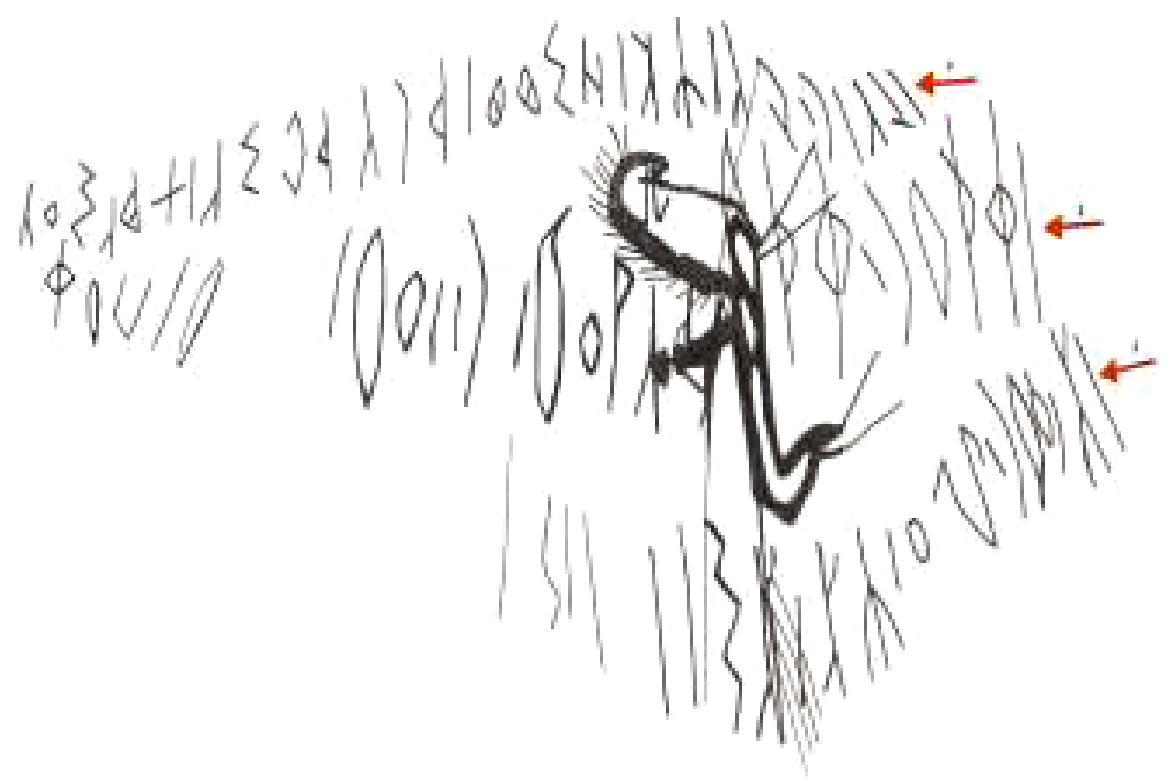




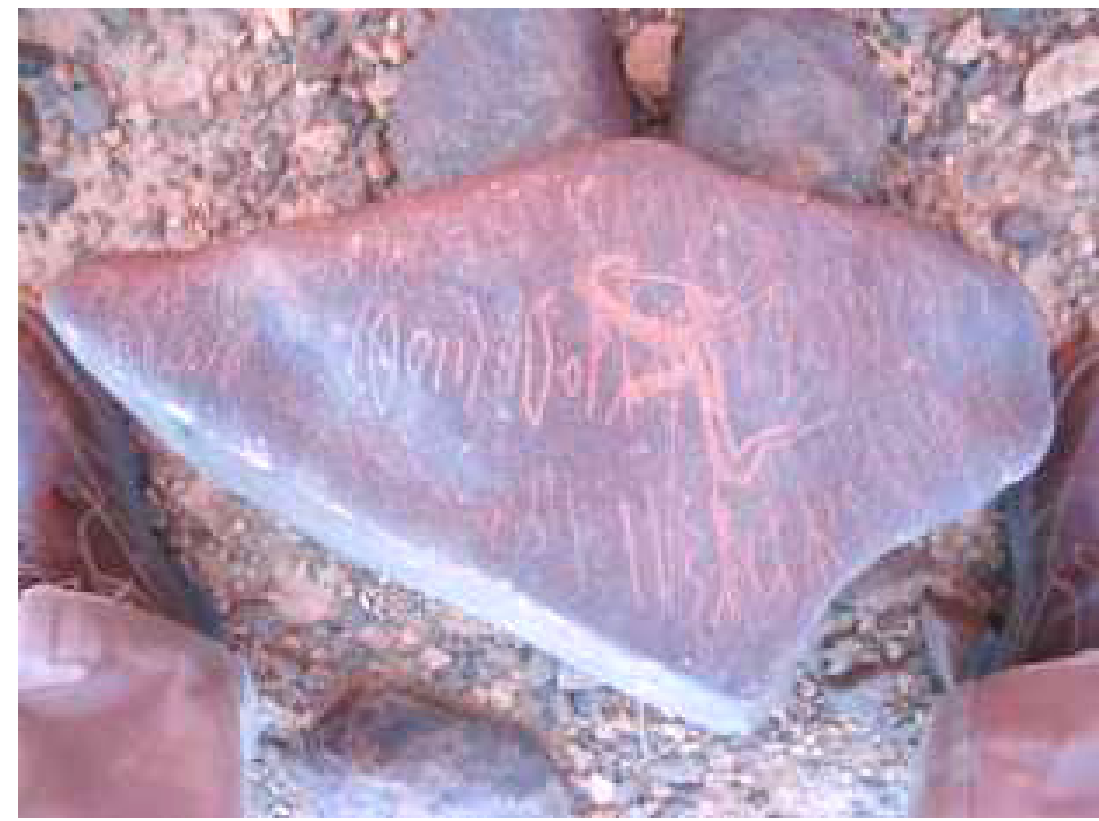

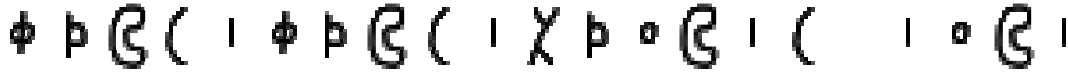

\section{Transliteration}

l qdm bn qdm bn 'd'mn bn n'mn

Von Qdm sohn von Qdm sohn von 'd'mn sohn von N'mn

Qdm: Littmann hat den Namen Qadam, Qudam, Qudaim oder Qädim vokalisiert, im Sinne von „high rank, bravery“ (LP 341). Der Name erscheint im Safaitischen (CIS 55; LP 28; SIJ 22), im Tamūdischen (King 1990, S. 536), im Nabatäischen in der Form Qdmw (PNNC 157), im Hatrenischen in der Form Qdm' hw[hy]


now 1930, S. 60) und im Arabischen Qadam, Qādim, Qudam und Qadìm (Gamharat an-Nasab 454 in Caskel 1966; PNHI 82).

'd'mn: Bei diesem Namen liegt wahrscheinlich eine 'f'l-Form von der Wurzel $d$ ' $m$, di 'ām „Unterstützung“, di 'ām „Stütze, Herr“ (Lane 1863-1893, S. 882) mit der Endung - $n$ vor. Der Name erscheint im Safaitischen (WH 2131; SIJ 729).

N'mn: Bei diesem Namen liegt eine Ableitung von der Wurzel $n$ ' $m$ mit der Endung $\bar{a} n$ vor, die möglicherweise als fa lān-Bildung zu vokalisieren ist. Vergleiche hierzu im Arabischen na ìm ,Wohlleben“. Der vorliegende Name erscheint im Safaitischen (KRS 5), im Qatabānischen (PQI 251), Lihyanisch (Caskel 1954, S. 150). Das Substantiv $N^{\prime} m$,Glück, Wohltat" erscheint sehr häufig im Safaitischen (CIS 96; LP 733), im Tamūdischen (King 1990, S. 637), im Qatabāni- 
schen (PQI 249-250), im Palmyrenischen (Stark 1971, S. 99) und an-Nu'mān im Arabischen (Gamharat an-Nasab 450 in Caskel 1966). In griechischer Transkription sind die von der gleichen Wurzelabgeleiteten Namen N $\alpha \alpha \mu \omega v$ und

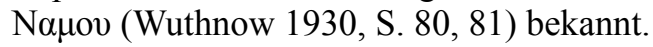

\section{Inschrift 3.}

\section{$\mid X 1 \cdot B(1 \Lambda \cdot 1 \lambda . X|||| \mid$}

\section{Transliteration}

l'n'm bn z'n $\underline{d}-' l s^{2} l l$

Von 'n 'm sohn von Z'n vom Stamme $S^{2} 11$

'n'm: Bei diesem Namen handelt es sich wohl um eine 'f' $l$-Bildung, 'an'am ,gnadenreich, gut, glücklich“, na'ama ,günstig sein, wohlhabend“ (Al-Manaser 2008, S. 118). Vergleichen lässt sich dazu: im Safaitischen (Voigt 1981, S. 180), im Tamūdischen (King 1990, S. 476; Shatnawi 2002, S. 652), im Lihyanischen (Caskel 1954, S. 107), im Minäischenen (PMI 66), im Nabatäischen (PNNC 40), im Palmyrenischen (Stark 1971, S. 70) und im Qatabānischen (PQI 88).

Z'n: Der Name könnte durch die arabischen Wörter $z \bar{a} \bar{a}^{\prime} a n a$, ,wandernd, reisend bzw. vergänglich“, za'ana ,gehen, laufen“, za'n „Aufbruch, Reise“, za ìn „Name des Kamels" (Lane 1863-1893, S. 1963) zu deuten sein. Vergleiche den Namen Z'n im Safaitischen (CIS 15; LP 237; HCH 196; SIJ 24; WH 28), im Tamūdischen (King 1990, S. 521), und im Nabatäischen in der Form T' $n w$ (Negev 1991, S. 33).

d-'l ( auch nur ' $l$, die Zugehörigkeit zu einem Stamm. Der Ausdruck steht vor dem Stammesnamen und folgt dem Personennamen bzw. der Genealogie (Al-Jallad 2015, S. 187).

$\mathbf{S}^{2}$ II: Es handelt sich um einen Stammesnamen, der hier zum ersten Mal in den safaitischen Inschriften erscheint. Der Stammesname erscheint auch als Personenname in den safaitischen Inschriften (CIS 648; LP 780; HCH 188; SIJ 335; WH 192), im Tamūdischen (King 1990, S. 516), im Lihyanischen (Caskel 1954, S. 90), im Sabäisch (HIn 355), und Šalal, Śalāl, Šallāl, Šulail und Šelīl im Arabischen (Gamharat an-Nasab 525 in Caskel 1966). 


\section{Inschrift 4.}

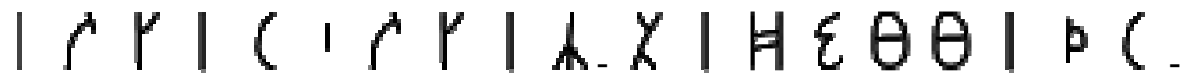 $r . P C E Y|+\theta r \circ Y \phi B \cap| B$}

\section{Transliteration}

l khl bn khl $\underline{d}-'$ 'l $d f w$ wld b-h-drfh lt $w h s^{2} h q m s^{1} l m$

Von Khl bin Khl vom Stamme Df. Und er brachte [die Tiere] in dieser Lagerstätte hervor. Und, o Lāt und $\mathrm{S}^{2}$ ai ' haq-Qaum, [gib] Heil.

Khl: $k a \overline{h i l}$, „kräftig, stark, bedeutender Mann, Held“, kahl ,im besten Mannesalter stehend, reifer, erwachsener Mann“ (Al-Manaser 2008, S. 238). Vergleicheden Namen außerdem im Safaitischen (KRS 23), im Tamūdischen (King 1990, S. 541), im Minäischen (PMI 154), im Lihyanischen (HIn 506), Khylw und Khlw im Nabatäischen (Negev 1991, S. 35), Khylw im Palmyrenischen (Stark 1971, S. 92),

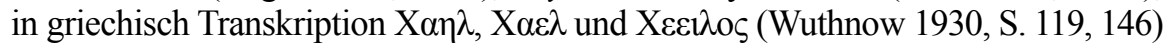
und Kāhil und Kahl im Arabischen (Gamharat an-Nasab 368 in Caskel 1966).

Df: Es handelt sich um den berühmtesten Stammesnamen in den safaitischen Inschriften (KRS 814; Al-Jallad 2015, S. 44).

b: $b i$ - Präposition ,,an, bei, in“ (Rawan 2013, S. 38).

wld: walada ,er brachte hervor“ (Lane 1863-1893, S. 3027; LP 1064; SIJ 281). Dieses Verb kommt meistens in den safaitischen Inschriften in der Verbindung mit dem Wort Ziege vor. In dieser Inschrift wissen wir leider nicht, welche Art von Tier er in dieser Lagerstätte hervorgebracht hat. Um die Inschriften zu verstehen und zu deuten, muss Umgebung der Fundorte der Inschriften näher betrachtet werden. Ein Beispiel dafür ist das Verb banaya mit der Bedeutung ,er baute“, w bny $h$ - $q b r$,er baute dieses Grab“. Man entdeckt dieses Verb in den Inschriften immer auf einem Stein, der auf einem Steinhügel liegt. Der Schreiber dieses Verbs will zeigen, dass er diesen Steinhügel oder dieses Grab gebaut hat. Ein anderes Beispiel ist das Verb ra'aya „er hütete, graste, weidete“. Der Schreiber meinte damit: „er hüteteden Platz“. Das Verb findet sich an offenen Plätzen und wo offenkundig ist, dass derVerfasser dort gehütet hat. Der Fundort der Inschriften kann wichtige Hinweise beider Analyse der Inschriften selbst liefern. Die Berücksichtigung dieser offensichtlichen Beziehung ist unabdingbar, um die wahre Bedeutung und den tatsächlichen Inhalt der Inschriften bei der Lesung zu erfassen.

h-dr: had-dawra „diese Lagerstätte“ (Al-Jallad-Al-Manaser 2015, S. 55).

$\mathbf{S}^{\mathbf{2}}$ '-h-qm: $\mathbf{S}^{\mathbf{2}}$ ai '-haq-qaum Einem der bekanntesten safaitischen Götter oblag wahrscheinlich der Schutz der Reisenden in den Karawanen (KRS 15, 18).

$\mathbf{s}^{1} \mathbf{l m}: s^{1} l m$ ist entweder Nominalform salām „Heil!“", „Sicherheit und Friede!“" oder als Imperativ sallim ,gib Heil““ zu deuten (WH 638; KRS 1). 
Inschrift 5.

EINIGE ALTNORDARABISCHE INSCHRIFTEN AUS JORDANIEN

465

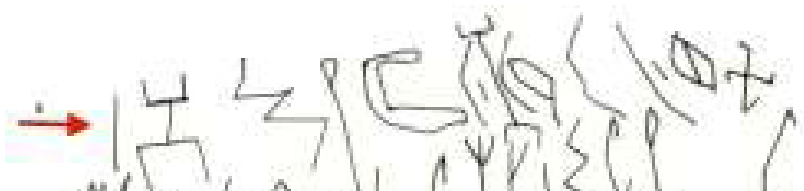

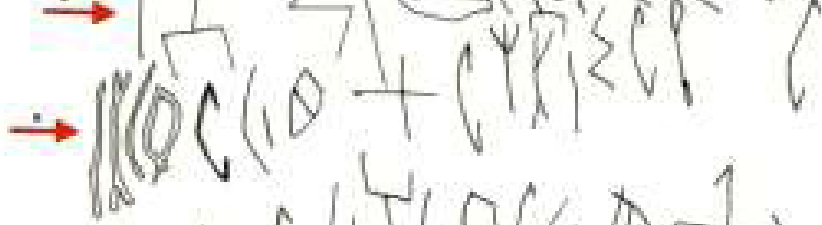

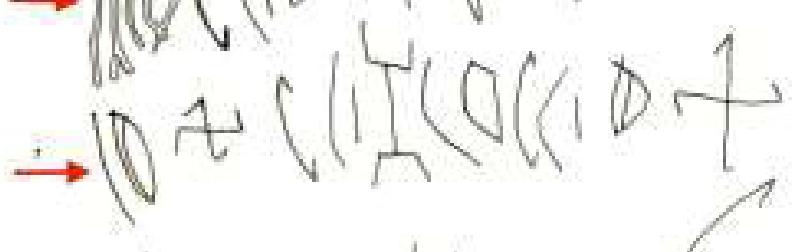

$$
\begin{aligned}
& \rightarrow \mathrm{O}\left(\mathbb{E}^{\mathrm{T}}\right. \\
& \text { ᄀ } 7 \text { (1) }
\end{aligned}
$$

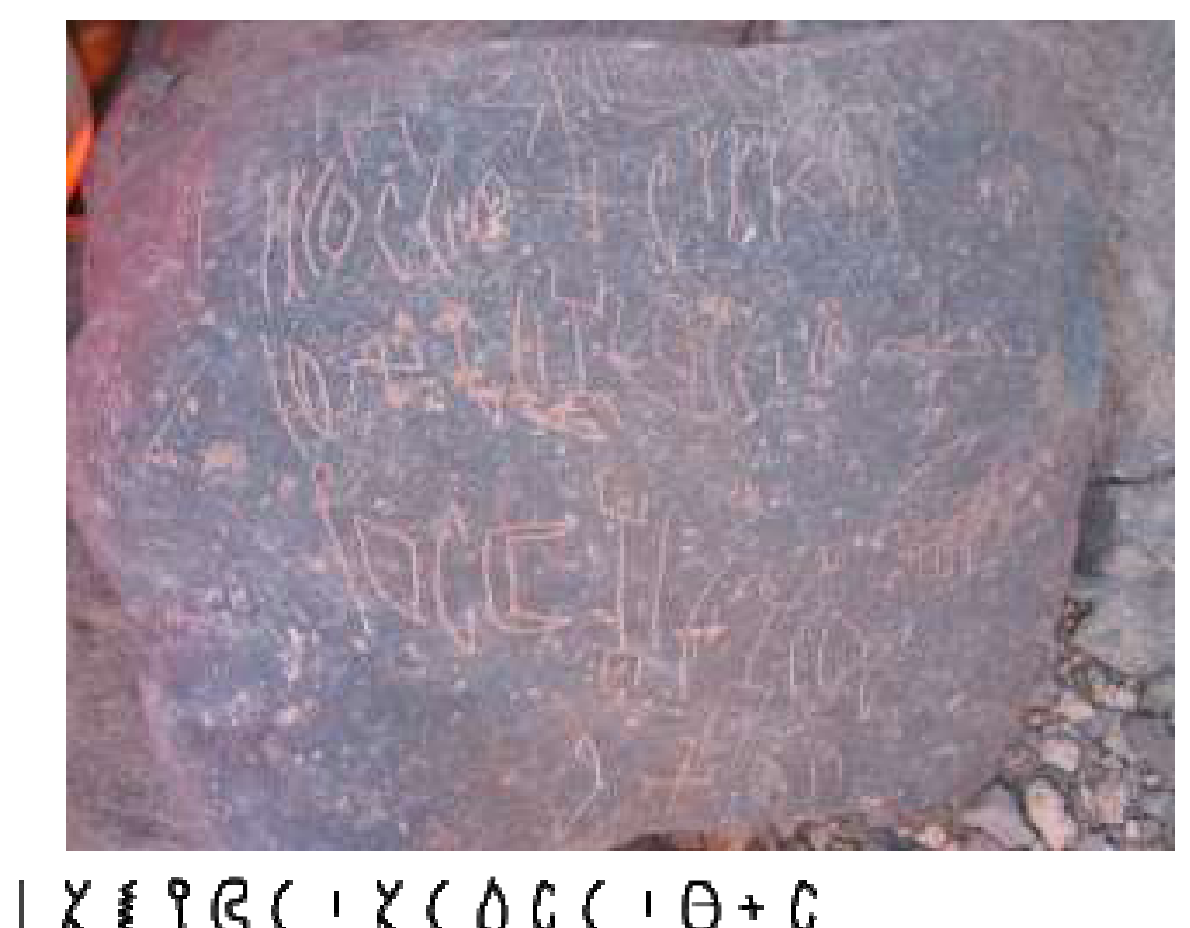

$|x| 9 \sec 1 \times \operatorname{coc}(1 \theta+C$

Acta Orient. Hung 69, 2016 


\section{Transliteration}

l's ${ }^{2} y m$ bn 'bgr bn wtr

Von 's ${ }^{2} \mathrm{ym}$ bin 'bgr bin Wtr

's $\mathbf{s}^{\mathbf{2} y m}$ : Bei dem Namen 's2ym handelt es sich wahrscheinlich um die Wurzel šym, arabisch šiyam „Natur, Veranlagung“ (Lane 1863-1893, S. 1644), sabäisch šym „einsetzen, errichten (Stele, Altar)“, akk. šâmu „festsetzen“ (Sholan 1999, S. 149). Der Name könnte 'Ašyam lauten. Damit zu vergleichen sind die im Folgenden genannten Namen: im Safaitischen (CIS 84; LP 162), $S^{2} y m$-' $l$ im Lihyanischen (Caskel 1954, S. 152), im Minäischen in der Form Yšym und Y̌sm $m$ (PMI 128) und 'Ašyam im Arabischen (Gamharat an-Nasab 201).

'bgr: Der nach der ' $f$ ' $l$-Form gebildete Name gehört zur Wurzel $b g r$, vergleiche arabisch 'abgar ,dickbäuchig“, 'abgara ,reich werden“(Lane 1863-1893, S. 153). Vergleiche im Safaitischen (LP 26), und Lihyanisch (Sima 1999, S. 54), im Nabatäischen die Formen 'bgrw und 'bgr (Negev 1991, S. 9), im Palmyrenischen (Stark 1971, S. 63), im Minäischen (PMI 188) und in griechischer Transkription 'A $\beta \gamma \alpha \rho o \varsigma$ (Wuthnow 1930, S. 7, 123).

Wtr: Die Deutung dieses Namen stützt sich wahrscheinlich auf die arabische Wurzel wtr, watīr „überragend“, watār ,übermäßig, überschüssig sein“ (Lane 18631893, S. 2978). Der Name dürfte Watīr zu vokalisieren sein. Vergleiche den Namen hierzu im Safaitischen (KRS 2285), im Tamūdischen (TIJ 73; King 1990, S. 560), im Lihyyanischen (Sima 1999, S. 81), Wtrm im Qatabānischen (PQI 264), Wtrw und Wtyrw im Nabatäischen (Negev 1991, S. 25) und Watīr und Watār im Arabischen (PNHI 95).

\section{Inschrift 6.}

\section{$\mid x \operatorname{coc}(1 \theta+G A . X \mid \varepsilon G !$}

\section{Transliteration}

\section{l'bgr bn wtr $\underline{d}$-'l frt}

Von 'bgr bin Wtr vom Stamme Frt

Es scheint, dass der Inhaber dieser Inschrift die gleiche Person wie in der Inschrift KRS 2285 ist.

Frț: Der Name eines safaitischen Stammes, der mehr als 25mal in den safaitischen Inschriften erscheint, wurde in verschiedenen safaitischen Inschriften aus Syrien und aus Jordanien gefunden (HCH 108; SIJ 241; Rawan 2013, S. 324). 


\section{Inschrift 7.}

\section{$\theta+C(1) \operatorname{coc}(1 \theta+c$}

\section{Transliteration}

lwtr bn'bgr bn wtr

Von Wtr bin 'bgr bin Wtr

\section{Inschrift 8.}

\section{$10 C B \times 1 C 1 \times C O C(1 \theta+C$}

\section{Transliteration}

lgrm'l bn 'bgr bn wtr

Von Grm'l bin'bgr bin Wtr

Es scheint, dass der Inhaber dieser Inschrift die gleiche Person wie in der Inschrift KRS 2748 ist $^{2}$.

Grm-'I: Hierbei handelt es sich um einen Personennamen aus der Wurzel grm und demth. El. 'l, Garm-'il „Erhabenheit Gottes“ oder „Entscheidung bzw. Wille Gottes“ oder „Gott hat entschieden“ (Hazim 1986, S. 22). Der Name Grm-'l ist wahrscheinlich das häufigste theophore Element im Safaitischen. Für Parallelen vergleiche im Safaitischen (CIS 242; SIJ 7), im Tamūdischen (Shatnawi 2002, S. 666), im Nabatäischen (Negev 1991, S. 19), im Lihyanischen (Caskel 1954, S. 146) und im Minäischen (PMI 81).

\section{Abkürzungsverzeichnis zur Literatur}

CIS Ryckmans, G. (ed.) (1950-1951): Corpus Inscriptionum Semiticarum. Pars V. Inscriptiones Saracenicas Continens, Tomus 1. Inscriptiones Safaiticae. Paris, Imprimerie nationale.

HCH Harding (1953, Plates 1-7).

HIn Harding (1971).

ISB Oxtoby (1968).

${ }^{2}$ KRS 2748: l grm'l bn 'bgr bn wtr $\underline{d}$ - 'l frt $\underline{w} w r$ ' $y$ h- ${ }^{\prime}$ 'n fh lt sllmw wqyt $m$ - $s^{2} n$ '.

Translation: By Grm'1 son of 'bgr son of Wtr of the lineage of Frt and he pastured the sheep and so O Lt [grant] security and protection from enemies. 
KRS "King Rescue Survey". Inscriptions recorded by Geraldine King on the Basalt Rescue Desert Survey in north-eastern Jordan in 1989. Now published in: The Online Corpus of the Inscriptions of Ancient North Arabia Project at the Khalili Research Centre, University of Oxford (http://krc.orient.ox.ac.uk/ociana/index.php).

LP Littmann (1943)

OCIANA The Online Corpus of the Inscriptions of Ancient North Arabia Project at the Khalili Research Centre, University of Oxford (http://krc.orient.ox.ac.uk/ociana/index.php)

PIH Abbadi (1983).

PMI Al-Said (1995).

PNHI Abdallah (1975).

PNNC Al-Khraysheh (1986).

PNPI Stark (1971).

PQI Hayajneh (1998).

SIJ Winnett (1957).

TIJ Harding-Littmann (1952).

WH Winnett-Harding (1978).

\section{Bibliographie}

Abbadi, S. (1983): Die Personennamen der Inschriften aus Hatra. Hildesheim, Olms (Texte und Studien zur Orientalistik 1.).

Abbadi, S. (2006): Nuqū̌s șafawiyya gadīdah min wād̄̄ salmā (al-bādiyyah al-'urduniyyah). Amman, Jordan University.

Abdallah, M., (1975): Die Personennamen in al-Hamdānī's al-Iklīl und ihre Parallelen in den altsüdarabischen Inschriften. Ein Beitrag zur jemenitischen Namengebung. Tübingen, Dissertationsdruck, Eberhard-Karls-Universität.

Al-Jallad, A. (2015): An Outline of the Grammar of the Safaitic Inscriptions. Leiden, Brill.

Al-Jallad, A.-Al-Manaser, A. (2015): New Epigraphica from Jordan I: A Pre-Islamic Arabic Inscription in Greek Letters and a Greek Inscription from North-eastern Jordan. Arabian Epigraphic Notes No. pp. 1, 51-70. Published online: 19 May 2015.

Al-Khraysheh, F. (1986): Die Personennamen in den nabatäischen Inschriften des Corpus Inscriptionum Semtiticarum. Marburg/Lahn, Dissertationsdruck, Philipps-Universität.

Al-Manaser, A. (2008): Ein Korpus neuer safaitischer Inschriften aus Jordanien. Aachen, Shaker Verlag (Semitica et Semitohamitica Berolinensia 10).

Al-Said, S. F. (1995): Die Personennamen in den minäischen Inschriften: Eine etymologische und lexikalische Studie im Bereich der semitischen Sprachen. Wiesbaden, Harrassowitz Verlag (Akademie der Wissenschaften und der Literatur, Mainz, Veröffentlichungen der Orientalischen Kommission 41).

Caskel, W. (1954): Lihyan und Lihyanisch. Köln-Opladen, Westdeutscher Verlag (Arbeitsgemeinschaft für Forschung des Landes Nordhein-Westfalen, Geistwissenschaften, 4.).

Caskel, W. (1966): Gamharat an-nasab: Das genealogische Werk des Hišām ibn Muhammad alKalbī. Bd. II. Leiden, Brill.

Harding, G. L. (1953): The Cairn of Hani. Annual of the Department of Antiquities of Jordan Vol. 2, pp. 8-56, Pls $1-7$.

Harding, G. L. (1971): An Index and Concordance of Pre-Islamic Arabian Names and Inscriptions. Toronto, University of Toronto Press (Near and Middle East Series 8). 
Harding, G. L. - Littmann, E. (1952): Some Thamudic Inscriptions from the Hashemite Kingdom of Jordan. Leiden, Brill.

Hayajneh, H. (1998): Die Personennamen in den qatabānischen Inschriften: Lexikalische und grammatische Analyse im Kontext der semitischen Anthroponomastik. Hildesheim, Georg Olms Verlag (Texte und Studien zur Orientalistik 10.).

Hazim, R. (1986): Die Safaitischen theophoren Namen im Rahmen der gemeinsemitischen Namengebung. Inaugurat Dissertation zur Erlangung der Doctorwürde des Fachbereichs Aussereuropäische Sprachen und Kulturen der Philipps-Universität Marburg / Lahn.

King, G. (1990). Early North Arabian Thamudic E: A Preliminary Description Based on a New Corpus of Inscriptions from the Hisma Desert of Southern Jordan and Published Material. (Unpublished $\mathrm{PhD}$ thesis.) School of Oriental and African Studies, London.

Lane, E. W. (1863-1893): An Arabic-English Lexicon. 8 vols. London, Willams and Norgate.

Littmann, E. (1943): Safaitic Inscriptions, Syria. Leiden, Brill (Publications of the Princeton University Archaeological Expeditions to Syria in 1904-1905 and 1909. Division IV, Section C).

Macdonald, M. C. A. (2004): Ancient North Arabian. In: Woodard, Roger D. (ed.): The Cambridge Encyclopedia of the World's Ancient Languages. Cambridge, Cambridge University Press, Chapter 16, pp. 488-533.

Macdonald, M. C. A. (2006): Burial between the Desert and the Sown: Cave-Tombs and Inscriptions near Dayr Al-Kahf in Jordan. Damaszener Mitteilungen Bd. 15, pp. 273-301, Pls 4254.

Negev, A. (1991): Personal Names in the Nabatean Realm. Jerusalem, Hebrew University of Jerusalem (Qedem: Monographs of the Institute of Archaeology, Hebrew University of Jerusalem, 32).

Oxtoby, W. G. (1968): Some Inscriptions of the Safaitic Bedouin. New Haven, American Oriental Society (American Oriental Series 50).

Rawan, Sch. (2013): Neue Safaitische Inschriften aus Süd-Syrien. Aachen, Shaker Verlag (Semitica et Semitohamitica Berolinensia 16.).

Shatnawi, M. A. (2002): Die Personennamen in den tamudischen Inschriften. Eine lexikalisch grammatische Analyse im Rahmen der gemeinsemitischen Namengebung. Ugarit-Forschungen Bd. 34, pp. 619-784.

Sholan, A. (1999): Frauennamen in den altsüdarabischen Inschriften. Hildesheim, Georg Olms Verlag (Texte und Studien zur Orientalistik, Bd. 11.).

Sima, A. (1999): Die Lihyanischen Inschriften von al- ${ }^{c} U a y b$ (Saudi-Arabien). Rahden/Westf., Marie Leidorf (Epigraphische Forschungen auf der Arabischen Halbinsel, 1.).

Stark, J. K. (1971): Personal Names in Palmyrene Insciptiones. Oxford, Clarendon.

Voigt, R. M. (1981): Einige altnordarabische Inschriften. Zeitschrift des Deutschen Palästina-Vereins Vol. 97, No. 2, pp. 178-187.

Winnett, F. (1957): Safaitic Inscriptions from Jordan. Toronto, University of Toronto Press (Near and Middle East Series 2).

Winnett, F. V.-Harding, G. L. (1978): Inscriptions from Fifty Safaitic Cairns. Toronto, University of Toronto Press (Near and Middle East Series 9).

Wuthnow, H. (1930): Die semitischen Menschennamen in griechischen lnschriften und Papyri des vorderen Orients. Leipzig, Dietrich (Studien zur Epigraphik und Papyruskunde, Band I, Schrift 4). 